\title{
Autoimmune Limbic Encephalitis Associated with Glutamic Acid Decarboxylase Antibodies
}

\author{
Krishnan Balagopal ${ }^{1}$, Sreekumar Govinda Shenoy², Durllav Dutta ${ }^{3}$, Elizabeth Kurian ${ }^{4}$ \\ ${ }^{1}$ Department of Neurology, MOSC Medical College, Kolenchery, Kochi, Kerala, India, \\ ${ }^{2}$ Department of Neurosurgery, MOSC Medical College, Kolenchery, Kochi, Kerala, India. \\ 3, 4 Department of Radiology, MOSC Medical College, Kolenchery, Kochi, Kerala, India.
}

\section{INTRODUCTION}

Limbic encephalitis (LE) is a clinical syndrome characterized by the progressive development of neuropsychiatric symptoms including personality changes, memory loss followed by seizures, autonomic dysfunction, and involvement of the medial temporal lobes. ${ }^{1}$ Limbic encephalitis most commonly presents secondary to an autoimmune aetiology which can be further subclassified into autoimmune and paraneoplastic. Autoimmune LE can be classified according to the presence of autoantibodies into two categories.

The first one is associated with antibodies to intracellular neuronal antigens and the other with antibodies to cell surface antigens. ${ }^{2}$ Intracellular antigens include $\mathrm{Hu}$, Ma2, Collapsin response - mediator protein 5 (CRMP - 5) and cell surface antigens like the voltage-gated potassium channel (VGKC), N - methyl - D - aspartate receptor (NMDA) and others. The autoimmune form of LE is important because it can be treated with immunotherapy if detected early. We present a case of a patient with recurrent vertigo followed by progressive cognitive dysfunction and involuntary movements. She was diagnosed with autoimmune limbic encephalitis associated with glutamic acid decarboxylase (GAD) 65 antibodies. Her symptoms showed improvement following immunomodulatory therapy.

\section{PRESENTATION OF CASE}

This 61-year-old female patient with no comorbidities presented to the outpatient department with a 3-week history of progressive unsteadiness on walking along with decreased sleep and irrelevant talk. She had worsening of symptoms over the last 2 days with decreased speech output, stiffness of all four limbs and involuntary movements of left upper limb. Clinical examination revealed drowsy patient with a GCS of 12 / 15, slow eye movements and dysarthria. There was generalised rigidity seen in all 4 limbs along with intermittent dystonic posturing of the left upper limb. There was no neck stiffness or meningeal signs. Vitals were within normal limits. Considering the progressive cerebellar involvement, cognitive decline, extrapyramidal involvement in the form of rigidity and limb dystonia, a possible autoimmune encephalitis was considered more likely. Routine blood investigations done were within normal limits. Antithyroid antibodies were normal as was an ultrasound of the abdomen. CSF testing was done which showed elevated CSF proteins ( $55 \mathrm{mg} / \mathrm{dl}$ ) with normal sugar and cells. Viral markers were negative in the CSF. Serum for autoimmune encephalitis and paraneoplastic markers were sent, which was negative. Serum for GAD - 65 antibody was sent considering the cerebellar ataxia and limb stiffness. GAD antibodies were found to be significantly elevated 3087 IU / ML (less than 10 IU / ML Normal Range).
Corresponding Author: Dr. Krishnan Balagopal, Consultant Neurologist, MOSC Medical College, Kolenchery, Kochi, India. E-mail: krishnan.balagopal@gmail.com

DOI: $10.14260 / \mathrm{jemds} / 2021 / 435$

How to Cite This Article:

Balagopal K, Shenoy SG, Dutta D, et al. Autoimmune limbic encephalitis associated with glutamic acid decarboxylase antibodies. J Evolution Med Dent Sci 2021;10(28):2131-2133, DOI: $10.14260 / j e m d s / 2021 / 435$

Submission 07-03-2021, Peer Review 12-05-2021, Acceptance 18-05-2021, Published 12-07-2021.

Copyright (@) 2021 Krishnan Balagopal et al. This is an open access article distributed under Creative Commons Attribution License [Attribution 4.0 International (CC BY 4.0)] 
Imaging done by MRI Brain with contrast that showed areas of diffusion restriction in the bilateral caudate nuclei, right putamen, and parasagittal regions of right frontal lobe along with subcortical and gyral FLAIR hyperintensities in the anterior frontal lobe, hippocampal and mammillary bodies and bilateral parieto-occipital lobes (Figures 1, 2, 3, 4). Meningeal enhancement and hyperperfusion were seen in the posterior fossa.

Findings were felt to be in keeping with a possible autoimmune limbic encephalitis. EEG done showed diffuse bihemispheric slow waves in keeping with the diagnosis. CT Thorax and Ultrasound abdomen did not show any abnormalities.

In view of possible GAD associated limbic encephalitis, patient was started on immune modulation with pulse dose methylprednisolone $1000 \mathrm{mg}$ daily for 5 days followed by a tapering schedule of oral steroids. She was also given ceftriaxone, acyclovir and levetiracetam and was started on limb physiotherapy.

She had improvement in sensorium and limb dystonia but limb rigidity persisted. She subsequently developed recurrent episodes of autonomic instability with hypotensive episodes. She was planned for further immune modulation with Intravenous Immunoglobulin.

Due to financial constraints, the patient's attenders were not willing to continue with treatment and left against medical advice.

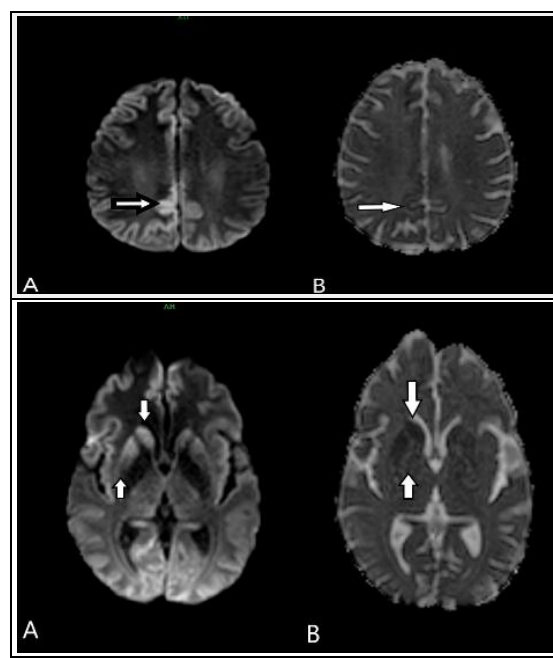

Figure 1.

A. Diffusion Restriction in Parasagittal Regions of Right Frontal Lobe B. Corresponding Areas on $A D C$

Figure 2.

A. Diffusion Weighted Imaging Showing Areas of Restricted Diffusion Involving Right Putamen and Caudate Nucleus $B$. Corresponding Areas on $A D C$

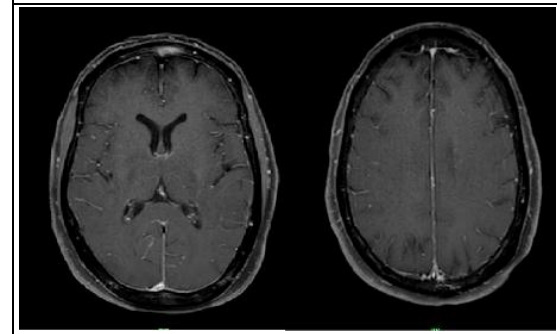

Figure 3.

Post Contrast Scan Showing Meningeal Enhancement, More in Posterior Regions

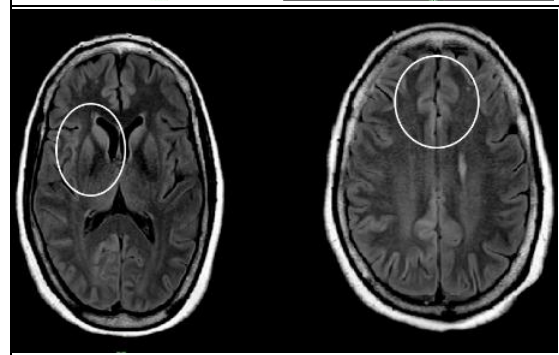

Figure 4.

FLAIR Sequences Showing Hyperintensities in Anterior Frontal Lobe, Parieto Occipital Region, Basal ganglia

\section{DISCUSSION}

There are numerous studies that show an association between autoimmune LE and the presence of GAD antibody. ${ }^{3}$ GAD antibodies are associated with a wide spectrum of neurological disorders. These include other conditions such as stiff-person syndrome (SPS), cerebellar ataxia, cognitive impairment, refractory seizures and oculo-palatal myoclonus. There is also an association between GAD antibody and anti GABA (B) receptor encephalitis which is frequently associated with small cell lung cancer and other lung tumours. ${ }^{4} \mathrm{GAD}$ is an enzyme which catalyses production of gamma aminobutyric acid (GABA). There are two isoforms of GAD antibody. GAD 67 is an enzyme present in the cytosol. It is distributed in the axonal processes and the dendrite filaments. GAD 65 is found predominantly in nerve terminals and seen anchored to neurotransmitter vesicles. The cofactor for GAD is PLP (Pyridoxal Phosphate) whose levels play a part in regulation of GAD activity. Around fifty percent of the GAD in the neurons is present as an apoenzyme form which can be readily converted to the active form as and when required. There are differences in the mechanism of activation of the isoforms of GAD. GAD 67 has been described to be inhibited by protein kinase A mediated phosphorylation. In contrast, GAD 65 is known to be inhibited by ATP mediated phosphorylation. Antibody formation has been postulated to take place due to exposure of the intracellular antigens during exocytosis. ${ }^{5}$ There is a dual mechanism of action. The first is a reduction in the synthesis of GABA while the second involves the inhibition of exocytosis of GABA. ${ }^{6}$ The most common syndrome associated with GAD antibodies is a form of Stiff Person Syndrome (SPS). Certain patients have limb rigidity along with other symptoms including stimulus sensitive myoclonus, gait unsteadiness, dysarthria and neuropsychiatric features. These are sometimes called SPS Plus syndromes. Limbic Encephalitis may belong to this spectrum of disorders. Compared to other forms of voltage gated LE, GAD antibody encephalitis tends to present at a slightly younger age. The two most common initial symptoms of presentation include refractory seizures and memory and cognitive disturbances. ${ }^{7}$ The clinical spectrum of Anti - GAD - associated encephalitis includes seizures (97\%), cognitive impairment (66\%), psychiatric symptoms (28\%), fever (14\%), dysautonomia (12\%), cerebellar features (7\%) and headaches (5\%). Seizures were the most common clinical features seen in these patients. Status epilepticus refractory to multiple anticonvulsants has been described. Memory and cognitive changes were the second most common syndrome found. These included frontal executive dysfunction, language disturbances, parietal lobe involvement in the form of various apraxias and presence of confabulation. Psychiatric features reported include depression, anxiety and occasionally disorders of personality. Severe dysautonomia has been reported as an important presenting symptom of anti - GAD related limbic encephalitis. One case report described death of a child with GAD antibody related encephalitis that occurred after immunotherapy. ${ }^{8}$ Rare symptoms reported include severe persistent headaches, facial cramps, increased appetite, oscillopsia and tinnitus. Sleep disorders and parasomnias have been described in rare cases. Gastrointestinal symptoms such as motility disorders and intestinal pseudo obstruction has been described in a few cases. 
MRI changes involving the medial frontal, temporal lobes, basal ganglia and cerebellum have been described in most cases. ${ }^{9}$ Changes have also been reported to be multifocal in some cases. EEG changes described slowing and epileptiform activity including focal sharp waves. A significant proportion of cases show positivity in both the serum and the CSF for GAD antibody. Coexistence of other antibodies has been reported in many cases. CSF abnormalities reported lymphocytic pleocytosis, raised proteins and presence of oligoclonal bands in some cases. Patients with GAD antibodies tend to respond less to immunotherapy as compared to the other cell surface antigens. Also, antibody titres do not correlate well with disease activity or response to treatment. Many patients do not improve immediately after immunotherapy, but may require a few months for symptoms to subside. This may be a reason for the poor response to treatment that has been reported in literature. Combination of treatments and repeated doses of the same may be required for a proper therapeutic response. Some patients may have residual neurological deficits due to irreversible cell death. Following stabilization of clinical response, there are reports of relapse following cessation of immunotherapy.

Intravenous steroid therapy is the initial treatment of choice in most cases. In cases that do not respond to therapy, immunoglobulin therapy and plasma exchange can be used. Immunoglobulin therapy is effective in these cases by modulating the action of $\mathrm{T}$ cells and cytokines. It may also accelerate the catabolism of systemically circulating immunoglobulins. Refractory cases may respond only to agents like cyclophosphamide and rituximab. ${ }^{10}$ These agents act by depletion of circulating B cells and may be associated with systemic side effects. Early and aggressive treatment may lead to complete remission of symptoms. Hence early detection of cases and prompt immunotherapy is of paramount importance in preventing severe and persistent neurological deficits.

\section{CONCLUSIONS}

Autoimmune limbic encephalitis is an emerging health threat in the general population which is frequently diagnosed late due to the heterogeneity of its clinical presentation. It can occur both in adults and in children. A wide variety of antibodies are associated, each with distinct clinical presentations. GAD antibody positive limbic encephalitis is a rare but important cause of limbic encephalitis that has to be considered in the differential diagnosis. This has to be kept in mind in adults presenting with cognitive decline, limb rigidity and dysautonomia. Prompt recognition of this condition and effective treatment may help the clinicians to avoid permanent cognitive and behavioural damage in the patients.

Financial or other competing interests: None.

Disclosure forms provided by the authors are available with the full text of this article at jemds.com.

\section{REFERENCES}

[1] Tuzun E, Dalmau J. Limbic encephalitis and variants: classification, diagnosis and treatment. Neurologist 2007;13(5):261-71.

[2] Honnorat J. Autoimmune limbic encephalitis: an expanding concept. Lancet Neurol 2010;9(1):24-5.

[3] Mata S, Muscas GC, Naldi I, et al. Non-paraneoplastic limbic encephalitis associated with anti-glutamic acid decarboxylase antibodies. J Neuroimmunol 2008;199(12):155-9.

[4] Lancaster E, Lai M, Peng X, et al. Antibodies to the GABA (B) receptor in limbic encephalitis with seizures: case series and characterisation of the antigen. Lancet Neurol 2010;9(1):67-76.

[5] Reetz A, Solimena M, Matteoli M, et al. GABA and pancreatic beta-cells: colocalization of glutamic acid decarboxylase (GAD) and GABA with synaptic-like microvesicles suggests their role in GABA storage and secretion. EMBO J 1991;10(5):1275-84.

[6] Vianello M, Tavolato B, Giometto B. Glutamic acid decarboxylase autoantibodies and neurological disorders. Neurol Sci 2002;23(4):145-51.

[7] Malter MP, Helmstaedter C, Urbach H, et al. Antibodies to glutamic acid decarboxylase define a form of limbic encephalitis. Ann Neurol 2010;67(4):470-8.

[8] Achour NB, Younes TB, Rebai I, et al. Severe dysautonomia as a main feature of anti-GAD encephalitis: report of a paediatric case and literature review. Eur J Paediatr Neurol 2018;22(3):548-5.

[9] Gagnon MM, Savard M. Limbic encephalitis associated with GAD65 antibodies: brief review of the relevant literature. Can J Neurol Sci 2016;43(4):486-93.

[10] Dalmau J, Gleichman AJ, Hughes EG, et al. Anti-NMDAreceptor encephalitis: case series and analysis of the effects of antibodies. Lancet Neurol 2008;7(12):1091-8. 